\title{
AN ALTERNATIVE PROOF OF A THEOREM ON UNIMODULAR GROUPS
}

MORRIS NEWMAN

Introduction. Let $G$ denote the multiplicative group of matrices

$$
\left(\begin{array}{ll}
a & b \\
c & d
\end{array}\right)
$$

where $a, b, c, d$ are integers and $a d-b c=1$, and $G_{0}(n)$ the subgroup of $G$ characterized by $c \equiv 0(\bmod n)$, where $n$ is an integer different from 0 . In a forthcoming paper [1] the author has proved the following theorem:

THEOREM 1. Let $H$ be a subgroup of $G$ containing $G_{0}(n)$. Then $H=G_{0}(m)$, where $m \mid n$.

The proof given was by an induction and made use of properties of the representatives of $G_{0}(n N)$ in $G_{0}(n)$. The referee for [1] furnished the author with an ingenious proof of Theorem 1 which avoided the induction. Since then the author has found a simpler proof which is more illuminating. This proof of Theorem 1 will be given here.

Set

$$
S=\left(\begin{array}{ll}
1 & 1 \\
0 & 1
\end{array}\right), \quad W=\left(\begin{array}{ll}
1 & 0 \\
1 & 1
\end{array}\right)
$$

We note that

$$
S^{k}=\left(\begin{array}{ll}
1 & k \\
0 & 1
\end{array}\right), \quad W^{k}=\left(\begin{array}{ll}
1 & 0 \\
k & 1
\end{array}\right)
$$

$S$ is an element of $G_{0}(n)$ for every $n$, and so $S \in H$.

LEMMA 1. Let

$$
M=\left(\begin{array}{ll}
a & b \\
c & d
\end{array}\right) \in H
$$

Then $W^{c} \in H$.

Proof. We have

$$
S^{x} M=\left(\begin{array}{ll}
1 & x \\
0 & 1
\end{array}\right)\left(\begin{array}{ll}
a & b \\
c & d
\end{array}\right)=\left(\begin{array}{cc}
a+x c & b+x d \\
c & d
\end{array}\right) \in H
$$

Received by the editors January 21, 1955. 
Since $(a, c)=1$, there is an $x$ such that $(a+x c, n)=1$. (This is a consequence of Dirichlet's theorem, but can be proved in an elementary fashion. See e.g., p. 17 [2].) Thus associated with every matrix

$$
M=\left(\begin{array}{ll}
a & b \\
c & d
\end{array}\right) \in H
$$

there is a matrix

$$
M_{0}=\left(\begin{array}{ll}
a_{0} & b_{0} \\
c & d
\end{array}\right) \in H
$$

such that $\left(a_{0}, n\right)=1$.

Since $\left(a_{0}, n\right)=1$, we can determine $y$ such that $a_{0} y \equiv c(\bmod n)$. Then

$$
W^{-y} M_{0}=\left(\begin{array}{rr}
1 & 0 \\
-y & 1
\end{array}\right)\left(\begin{array}{ll}
a_{0} & b_{0} \\
c & d
\end{array}\right)=\left(\begin{array}{cc}
a_{0} & b_{0} \\
c-a_{0} y & d-b_{0} y
\end{array}\right) \in G_{0}(n) .
$$

Hence $W^{-y} M_{0} \in H$, and so $W^{-\imath} \in H$. Thus $W^{a_{0} y} \in H$. Since $a_{0} y \equiv c$ $(\bmod n)$ and $W^{n} \in G_{0}(n) \subseteq H, W^{c} \in H$. Lemma 1 is thus proved.

Lemma 2. Let $Z$ denote the totality $\{c\}$, where

$$
\left(\begin{array}{ll}
a & b \\
c & d
\end{array}\right) \in H
$$

Then $Z$ is an ideal in the ring of integers and hence a principal ideal.

Proof. This is immediate, since if $c_{1}, c_{2} \in Z$ then $W^{c_{1}}, W^{c_{2}} \in H$ by Lemma 1, and so for arbitrary integers $p, q$

$$
\left(W^{c_{1}}\right)^{p}\left(W^{c_{2}}\right)^{q}=W^{c_{1}+q_{1} c_{2}} \in H,
$$

whence $p c_{1}+q c_{2} \in Z$.

We turn now to the proof of Theorem 1. Put $Z=(m)$. Since $G_{0}(n)$ $\subseteq H,(n) \subseteq Z$, and so $m \mid n$. Trivially, $H \subseteq G_{0}(m)$. Furthermore, let

$$
M=\left(\begin{array}{cc}
a & b \\
m c & d
\end{array}\right) \in G_{0}(m) .
$$

Reasoning as before, we can find an $x$ such that

$$
S^{x} M=\left(\begin{array}{ll}
a_{0} & b_{0} \\
m c & d
\end{array}\right)
$$

where $\left(a_{0}, n\right)=1$. Since $\left(a_{0}, n\right)=1$, we can determine $y$ so that $a_{0} y \equiv-1(\bmod n)$. For this $y$, 


$$
\begin{aligned}
W^{m c y} S^{x} M & =\left(\begin{array}{cc}
1 & 0 \\
m c y & 1
\end{array}\right)\left(\begin{array}{ll}
a_{0} & b_{0} \\
m c & d
\end{array}\right) \\
& =\left(\begin{array}{cc}
a_{0} & b_{0} \\
m c\left(a_{0} y+1\right) & m c y b_{0}+d
\end{array}\right) \in G_{0}(n) .
\end{aligned}
$$

Hence $W^{m c y} S^{x} M \in H$. But Lemmas 1 and 2 imply that $W^{m c y} \in H$. Since also $S^{x} \in H, M \in H$. Thus $G_{0}(m) \subseteq H$, and so $H=G_{0}(m)$. This completes the prosf of Theorem 1 .

\section{REFERENCES}

1. M. Newman, Structure theorems for modular subgroups, Duke Math. J., March 1955.

2. L. E. Dickson, Modern elementary theory of numbers, University of Chicago Press, 1943.

National Bureau of Standards 